IIIIIIIIIIIIIIIIIIIIIIIIIIIIIIIIIIII

Original Article

IIIIIIIIIIIIIIIIIIIIIIIIIIIIIIIIIII

\title{
Transcriptome analysis of Arabidopsis thaliana treated with green leaf volatiles: possible role of green leaf volatiles as self-made damage-associated molecular patterns
}

\author{
Yasuo Yamauchi, * Aya Matsuda, Nagisa Matsuura, Masaharu Mizutani and \\ Yukihiro Sugimoto \\ Graduate School of Agricultural Science, Kobe University, 1-1 Rokkodai, Nada-ku, Kobe 657-8501, Japan
}

(Received March 29, 2018; Accepted June 23, 2018)

\begin{abstract}
Green leaf volatiles (GLVs), which include C6 aldehydes, alcohols, and their esters, are emitted by damaged plants and are, therefore, thought to be involved in stress responses. However, the effects of GLVs on gene expression are not fully understood. Thus, the aim of the present study was to analyze the early transcriptional responses of Arabidopsis to the major GLVs-(Z)-3-hexenal, (Z)-3-hexenol, (E)-2-hexenal, and (Z)-3-hexenyl acetate-using comprehensive microarray gene expression analysis. All of the GLVs induced changes in gene expression, and (Z)-3-hexenal, (Z)-3-hexenol, and (Z)-3-hexenyl acetate commonly triggered the expression of defense-related genes, whereas $(E)$-2-hexenal mainly induced genes responsible for responding to abiotic stress, such as heat and oxidative stress. These results suggest that GLVs can function as airborne infochemicals that regulate the rapid expression of defense response-related genes and that GLVs might play a physiological role as self-made damage-associated molecular patterns (DAMPs) in damaged leaves. ㅇ Pesticide Science Society of Japan

Keywords: airborne infochemical, Arabidopsis, damage-associated molecular patterns, defense response, green leaf volatile, transcriptome.
\end{abstract}

Electronic supplementary material: The online version of this article contains supplementary material (Supplemental Fig. S1 and Supplemental Tables 1-4), which is available at http://www.jstage.jst.go.jp/browse/jpestics/

\section{Introduction}

Plants emit a variety of volatile organic compounds (VOCs), usually in response to stressful conditions, ${ }^{1)}$ especially wounding. Furthermore, green leaf volatiles (GLVs) constitute a major portion of the VOCs emitted in response to mechanical wounding, pathogenesis, and herbivory. Therefore, phytochemists have deeply considered GLVs' chemical, biochemical, and biological properties; as a result, GLVs have been chemically characterized as C6 aldehydes, alcohols, and their esters, which play a variety of biological roles. ${ }^{2)}$

GLVs are biosynthesized from linolenic acid, which is a major component of the thylakoid membrane (Fig. 1). ${ }^{3)}$ More specifically, dioxygen is added to linolenic acid at position 13 by lipoxygenase, producing linolenic acid 13-hydroperoxide, which is then cleaved by hydroperoxide lyase at the $\mathrm{C} 12-\mathrm{C} 13$ bond to produce (Z)-3-hexenal (3-Hal, 1). Following this step, GLV biosynthesis branches into (Z)-3-C6 and (E)-2-C6 pathways, de-

\footnotetext{
* To whom correspondence should be addressed.

E-mail: yamauchi@kobe-u.ac.jp

Published online August 7, 2018

(c) Pesticide Science Society of Japan
}

pending on the activity of $(Z)-3:(E)-2$-hexenal isomerase (HI). ${ }^{4)}$ 3 -Hal is reduced to $(Z)-3$-hexenol (3-Hol, 3) by reductases with NADPH as an endogenous reductant. ${ }^{5)}$ Candidate enzymes include aldehyde dehydrogenases, aldehyde reductases, and aldo/ keto reductases, but an enzyme essential for 3-Hal reduction has not yet been identified. 3-Hol is further converted to $(Z)$-3-hexenyl acetate (3-HAC, 4) by acetyl CoA:(Z)-3-hexenol acetyltransferase (CHAT), a BAHD acetyltransferase family protein, ${ }^{6}$ and if sufficient $\mathrm{HI}$ activity is present, 3 - $\mathrm{Hal}$ is converted to $(E)$ 2-hexenal (2-Hal, 2), which is then converted to 2-Hol following 2-hexenyl acetate with the same enzymatic mechanism involved in the (Z)-3-C6 series.

Reflecting the GLV biosynthesis pathway, the emission of each GLV occurs sequentially after wounding. In Arabidopsis thaliana, the first GLV to be emitted is $3-\mathrm{Hal}$, which reaches maximum emission at approximately $30-45 \mathrm{sec}$ after wounding, whereas peak $3-\mathrm{Hol}$ emission occurs at approximately $2.5 \mathrm{~min}$ after wounding, and peak 3-HAC emission occurs $4.5-5.5 \mathrm{~min}$ after wounding. ${ }^{6}$ This sequence of emissions is likely common among plant species, since it has also been observed in a woody plant, namely aspen, from which $3-\mathrm{Hal}$ is emitted 5 min after wounding, peak $2-\mathrm{Hal}$ emission occurs at $13 \mathrm{~min}$, peak hexenol emission occurs at $16 \mathrm{~min}$, and peak hexenyl acetate emission 


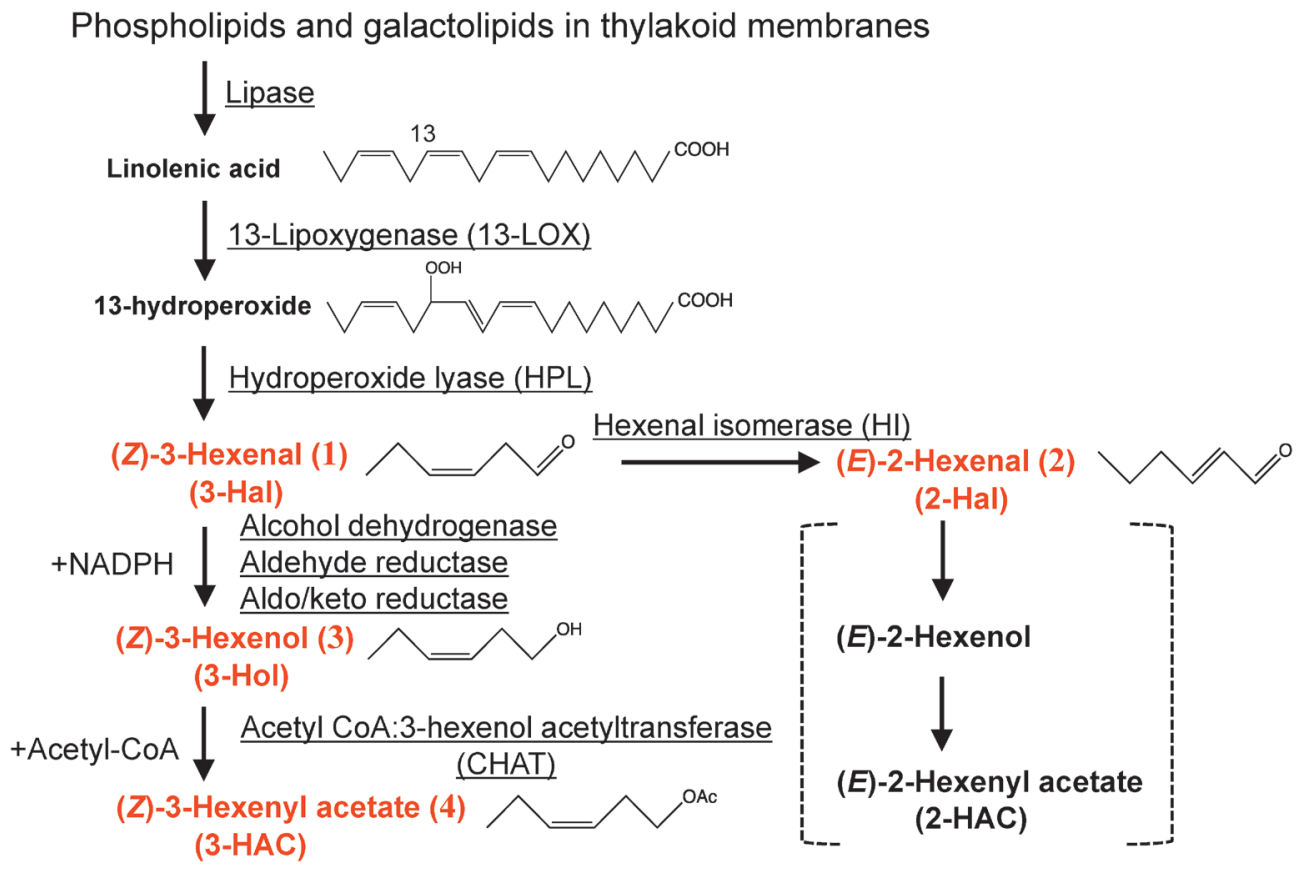

Fig. 1. Biosynthesis pathway of green leaf volatiles (GLVs). Enzymes are underlined, and red text indicates the GLVs used in the present study. (E)2-Hexenol and (E)-2-hexenyl acetate (dotted brackets) were not tested in the present study because they are relatively minor GLVs.

occurs at 34 min. $^{7)}$

Such rapid post-wounding GLV emission is thought to play an important role in defense responses, since GLVs possess direct defense properties, such as antibiotics ${ }^{8,9)}$ and insecticides. ${ }^{10,11)}$ In addition, GLVs function as airborne infochemicals mediating plant-insect, plant-herbivore, and plant-plant interactions. In plant-insect interactions, GLVs are emitted by herbivore-attacked leaves to attract the natural enemies of herbivores, thereby averting the crisis. ${ }^{12-14)}$ In plant-plant interactions, such as among tomato plants, 3-Hol is emitted by herbivore-infested plants and recognized by undamaged plants as a signal to form a defensive compound, e.g., 3-hexenylvicianoside, which possesses a defensive function against the common cutworm (Spodoptera litura) in tomato. ${ }^{15}$ ) These phenomena indicate that plants use GLVs to interact with organisms in the external environment.

Recent studies have supported the idea that plants use GLVs as endogenous infochemicals, i.e., GLVs are capable of inducing the expression of genes involved in responses to both biotic ${ }^{16)}$ and abiotic ${ }^{17)}$ stresses, and GLVs activate and prime plant defense systems against pathogen attack. ${ }^{18,19)}$ Furthermore, since GLVs are produced when plant tissues are damaged, the compounds can be categorized as damage-associated molecular patterns (DAMPs). ${ }^{20)}$ Therefore, the aim of the present study was to analyze the early transcriptional level responses of $A$. thaliana to four major GLVs (3-Hal, 3-Hol, 2-Hal, and 3-HAC) using comprehensive microarray gene expression analysis. As a result, we found that each GLV regulated the expression of distinct groups of genes and finally hypothesized that GLVs function as selfmade DAMPs.

\section{Materials and Methods}

\section{Chemicals}

(E)-2-Hexenal, (Z)-3-hexenol, and (Z)-3-hexenyl acetate were purchased from Tokyo Kasei Ltd. (Tokyo, Japan), whereas (Z)3-hexenal was a generous gift from Zeon Co. (Tokyo, Japan). Other reagents were purchased from Wako Pure Chemical Industries (Osaka, Japan) and Nacalai Tesque (Kyoto, Japan).

\section{Plant materials}

Arabidopsis thaliana (Columbia-0 ecotype, Col-0) seeds were sown on Jiffy-7 peat pellets (Sakata Seed Co., Yokohama, Japan) and incubated at $4^{\circ} \mathrm{C}$ in the dark for 3 day. The seedlings were then maintained at $23^{\circ} \mathrm{C}$ under a $14 \mathrm{hr}$ photoperiod $(80 \mu \mathrm{mol}$ photons $\mathrm{m}^{-2} \mathrm{~s}^{-1}$ ).

\section{Volatile treatment}

Volatile treatment was administered as described previously. ${ }^{17)}$ Briefly, Arabidopsis plants (4 weeks old) were placed in a transparent plastic box $\left(340 \mathrm{~cm}^{3}\right.$; Nippon Genetics, Tokyo, Japan), and acetonitrile $(\mathrm{MeCN})$-diluted volatile (total volume of $3 \mu \mathrm{L}$ ) was absorbed into a piece of paper towel attached to the inside of the lid in order to achieve a vapor density of $10 \mathrm{nmol} \mathrm{cm}^{-3}$ when the GLV had completely evaporated. Then, after covering tightly with the lid, the plants were incubated at $25^{\circ} \mathrm{C}$ for $30 \mathrm{~min}$ under illumination $\left(80 \mu \mathrm{mol}\right.$ photons $\left.\mathrm{m}^{-2} \mathrm{~s}^{-1}\right)$. MeCN-treated plants were used as controls.

\section{Microarray RNA sample preparation and hybridizations} Total RNA was purified from at least six plants of each treat- 
ment using an RNeasy Plant Mini Kit (Qiagen), and the quality of the RNA was confirmed using electrophoresis and spectrometric analysis in which both $\mathrm{A}_{260} / \mathrm{A}_{230}$ and $\mathrm{A}_{260} / \mathrm{A}_{280}$ ratios were higher than 1.8. Then Cy3-labeled cRNA was prepared using a one-color Low Input Quick Amp Labeling Kit (Agilent Technologies, Santa Clara, CA, USA), and microarray experiments were performed using an Agilent Arabidopsis ver4.0 (44k) microarray (Agilent Technologies), the one-color method, Gene Expression Hybridization Kit, and Gene Expression Wash Buffers Pack (Agilent Technologies), in accordance with the manufacturer's instructions.

\section{Microarray data evaluation and gene ontology analysis}

Raw data were evaluated using Agilent Feature Extraction software. Data extraction, normalization, and UPGMA (unweighted pair group method with arithmetic mean) clustering heat map production were performed using Subio Platform software (Subio Inc., Kagoshima, Japan), and the data were organized and interpreted using Microsoft Excel. Genes with values above 2.0 or below 0.5 were considered up- or downregulated, respectively. The reference gene expression datasets under each stressed condition were obtained using the AtGenExpress data- base (The Arabidopsis Information Resource; Ohio State University, Columbus, OH, USA). ${ }^{21)}$ Shoot expression data in time courses $(0.25,0.5,1,3$, or $6 \mathrm{hr}$ of heat, UV-B, drought, or wound stress as well as $0.5,1,3,6$, or $12 \mathrm{hr}$ of oxidative, salt, osmotic, or cold stress) are used for constructing the heat map. Up- and downregulated genes were listed using Subio Platform software, and gene ontology analysis was performed using DAVID version $6.8 .^{22)}$

\section{Results and Discussion}

\section{Arabidopsis thaliana distinguishes green leaf volatiles}

We previously performed a comprehensive analysis of gene expression in A. thaliana treated with 2-Hal using a NimbleGen Array and found that 2-Hal could function as an infochemical by inducing heat- and oxidative stress-responsive gene expression. ${ }^{17)}$ In the present study, we analyzed the effect of other GLVs on gene expression using the same experimental conditions and an Agilent microarray. To compare data obtained under identical experimental conditions, the gene expression analysis of 2-Hal-treated $A$. thaliana was performed again using an Agilent microarray.

In general, stress response genes were expressed in a correlat-

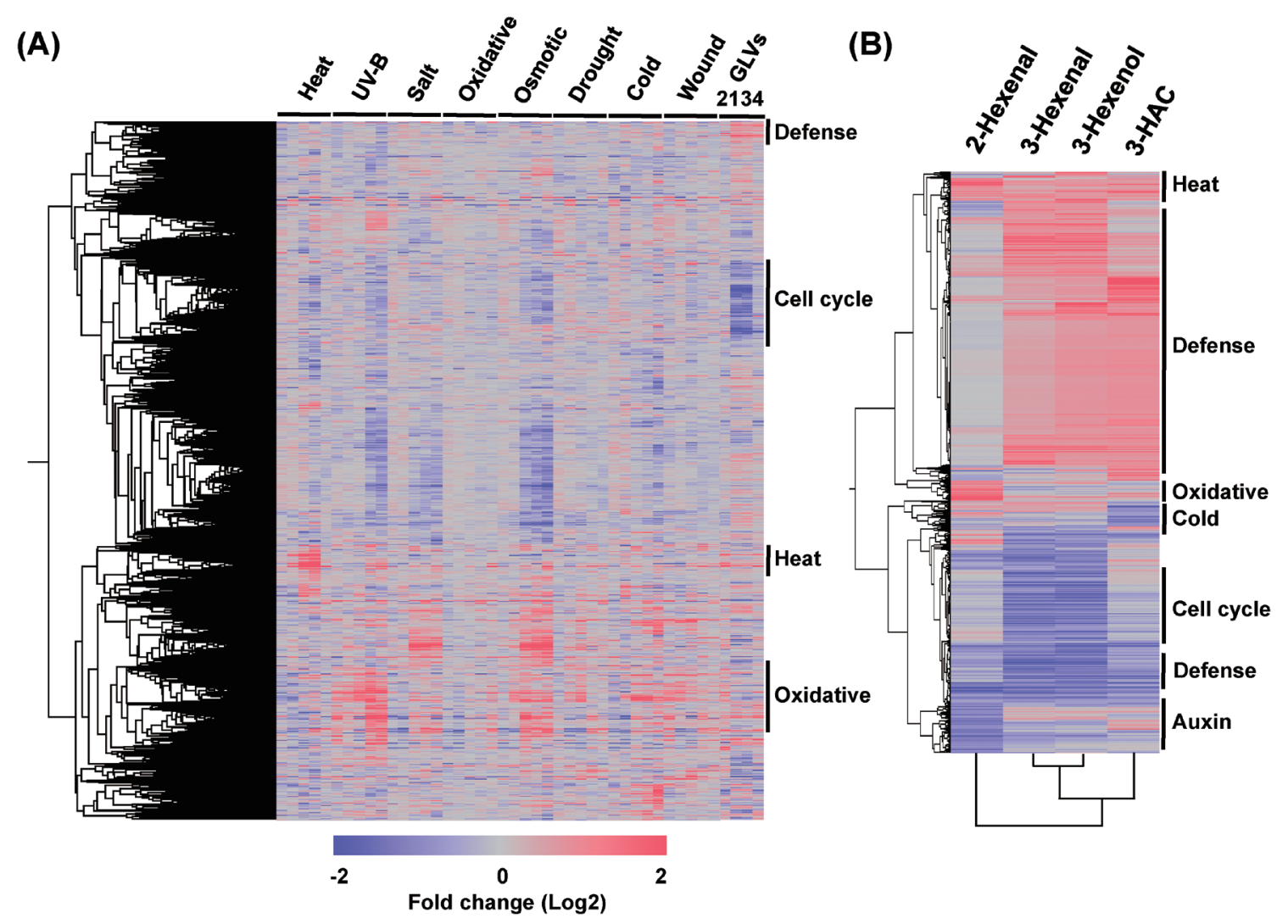

Fig. 2. Comprehensive gene expression analysis of A. thaliana treated with GLVs. (A) Heat map of whole gene expression by GLV-or stress-treated A. thaliana. Expression data were collected from shoots over two courses of treatment $(0.25,0.5,1,3$, or $6 \mathrm{hr}$ of heat, UV-B, drought, or wound stress as well as $0.5,1,3,6$, or $12 \mathrm{hr}$ of oxidative, salt, osmotic, or cold stress) obtained from the AtGenExpress database. ${ }^{21)}$ GLV treatments were administered using $(E)$ 2-hexenal (lane 2), (Z)-3-hexenal (lane 1), (Z)-3-hexenol (lane 3), and (Z)-3-hexenyl acetate (lane 4). (B) Selected heat map reconstituted from whole heat map shown in panel A. Only the genes upregulated $(>2)$ or downregulated $(1 / 2>)$ by at least one GLV are shown. Major biological terms extracted by gene ontology analysis are indicated on the right sides of the heat maps. 
ed and synchronized manner. UPGMA clustering analysis of the expression data obtained from 2-Hal-treated plants and various stressed samples suggested that 2-Hal synchronously induced heat- and oxidative stress-responsive genes. ${ }^{17)}$ Therefore, we used the same strategy for comprehensive analyses of the other GLVs, namely 3-Hal, 3-Hol, and 3-HAC. As a result, each GLV showed distinct gene expression patterns (Fig. 2).

The 2-Hal analysis also indicated that the upregulated genes were responsive to abiotic stresses such as heat and oxidative stresses, which corresponded to our previous results. On the contrary, a large portion of the genes regulated by $3-\mathrm{Hal}, 3-\mathrm{Hol}$, and 3-HAC was quite different from those regulated by $2-\mathrm{Hal}$, i.e., 3-Hal, 3-Hol, and 3-HAC mainly upregulated genes responsible for biotic defense response. Comparing the gene expression patterns induced by the four GLVs resulted in the classification of gene expression patterns into three types: 3-Hal and 3-Hol type, 2-Hal type, and 3-HAC type (Fig. 2B). These results suggest that $A$. thaliana can distinguish each GLV, probably due to independent response mechanisms; thus, each GLV induced distinct gene expression.

\section{Effects of 3-hexenal (3-Hal, 1) and 3-hexenol (3-Hol, 3) on gene expression}

Because 3-Hal is oriented at the most upstream position of GLV biosynthesis, 3-Hal is the first GLV emitted from wounded leaves (within $60 \mathrm{sec})^{6}$ ) and the most abundant GLV. ${ }^{5)}$ The rapid emission of 3-Hal is probably supported by the sufficient activities of enzymes involved in the emission of 3-Hal, i.e., lipase, lipoxygenase, and hydroperoxide lyase, in addition to their enzymatic properties for which they do not need coenzymes for reactions. Therefore, the first biological impact of GLV emission after wounding might depend on 3-Hal. However, gene expression profiles between wounding and 3 -Hal were quite different (Fig. 2A), indicating that the effect of 3-Hal on gene expression might be restricted to within the local area near a damaged site.

The 3-Hal treatment up- and downregulated 6419 and 4113 genes, respectively (Fig. 3A). Gene ontology analysis indicated that defense-related genes were up-regulated (Fig. 3B). For instance, PR2 was induced (ranked 8 th), which is a defense response marker gene of salicylic acid (SA), suggesting that the 3-Hal-induced defense response might be mediated by the SAresponsible pathway. 3-Hal also upregulated a number of receptor-like protein genes (Fig. 3B). Cysteine-rich receptor-like protein kinases (CRKs) are a type of pattern-recognition receptor (PRR) that is involved in plant immunity. ${ }^{23,24)}$ Even though plant immunity only involves innate immunity, plants are resistant to most microbes and pests due to a variety of PRRs for sensitive and rapid detection of their invasion. ${ }^{25)}$ Therefore, the induction of a number of PRRs might be involved in the priming effect against pathogens. It is worth noting that 3-Hal downregulated a number of genes, and gene ontology analysis indicated that genes downregulated by 3 -Hal treatment were involved in the cell cycle process, thereby indicating that lowering the fundamental processes of intact cells might be a pharmacological function of 3-Hal.

After the synthesis of 3-Hal, 3-Hol is produced through the reduction of 3-Hal, possibly by alcohol dehydrogenases, aldehyde reductases, or aldo/keto reductases. Because this reaction requires NADPH supplied by the integrated NADPH reduction system, intact cells should be the sites of 3-Hol production.

The 3-Hol treatment also affected gene expression in a manner similar to that of 3-Hal (Fig. 2B), with a high correlation factor $(r=0.920)$, as shown in Fig. S1. The number of genes downregulated by 3 -Hol $(n=4196)$ was almost equal to the number downregulated by 3 -Hal ( $n=4113$; Fig. $4 \mathrm{~A})$, although the number of genes upregulated by $3-\mathrm{Hol}(n=3396)$ was significantly lower than the number upregulated by $3-\mathrm{Hal}(n=6419)$. In addition, chitinase (At2g43570) was one of the genes most upregulated in both 3-Hal- and 3-Hol-treated A. thaliana, although the induction ratio of $3-\mathrm{Hol}$ (26.7-fold) was less than that of 3-Hal (79.3-fold). These results suggest that the effect of 3-Hol on the expression of defense response genes was weaker than that of 3-Hal.

As shown in Fig. 4B, defense response genes were upregulated; however, contrary to the results after 3-Hal treatment, several heat stress response genes were listed in the Top100 upregulated genes following 3-Hol treatment. An important transcription factor for heat stress response, HSFA2, was ranked 68th among the top 100 upregulated genes, which suggested that 3 -Hol is involved in both heat stress response (i.e., protein

(A)

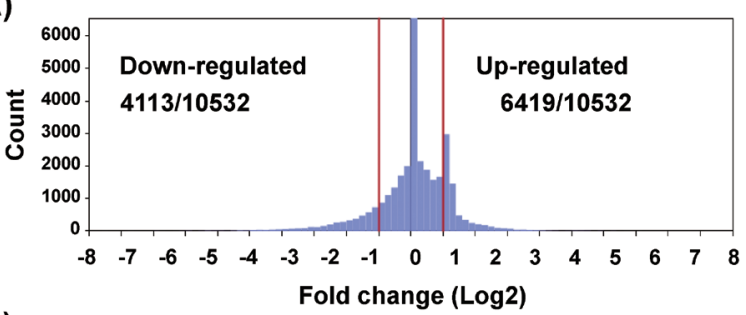

(B)

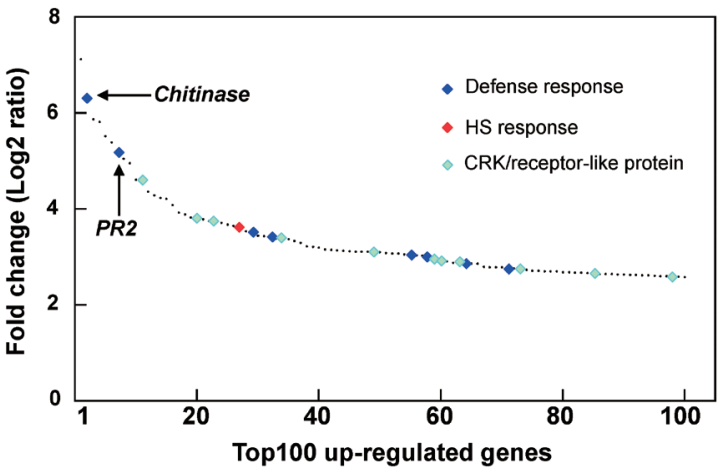

Fig. 3. Effect of 3-Hal on gene expression in A. thaliana. (A) Histogram of fold changes ( $\log 2$ ratio) in whole gene expression. Frequency of upregulated $(>2)$ and downregulated $(1 / 2>)$ genes per sum of up- and downregulated genes are shown in the graph. (B) The top 100 genes highly upregulated by 3 -Hal treatment. Triangles indicate defense response genes (blue), heat stress response genes (red), and cysteine-rich receptor and receptor-like protein genes (pale blue). Dots indicate other miscellaneous classes of genes. A detailed list is shown in Table S1. 
(A)

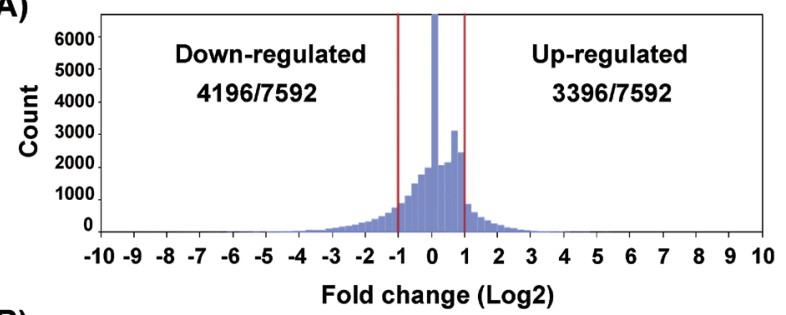

(B)

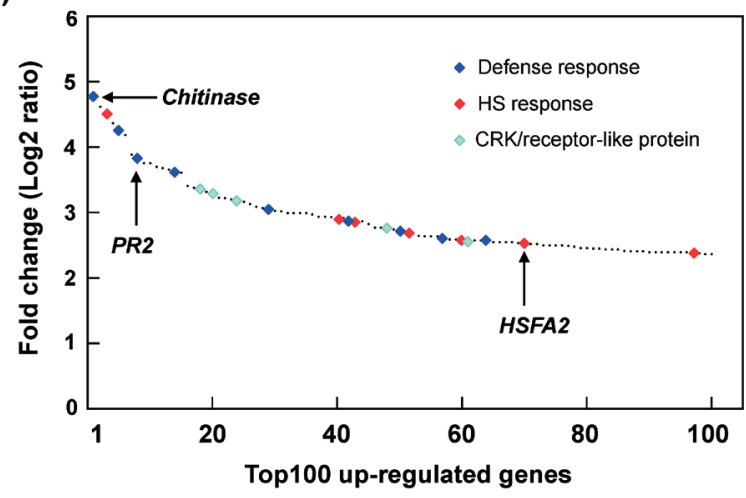

Fig. 4. Effect of 3-Hol on gene expression in Arabidopsis. Legends of panels (A) and (B) are the same as those for Fig. 3. A detailed list is shown in Table S2.

homeostasis) and defense response. Furthermore, 3-Hol also downregulated genes involved in the cell cycle process (Fig. 2B).

\section{Effects of 2-hexenal (2-Hal, 2) on gene expression}

2-Hal is biosynthesized by the (Z)-3:(E)-2-hexenal isomerasemediated isomerization of 3-Hal. ${ }^{4)}$ We previously reported that a wild-type tomato cultivar (Micro-Tom), which has undetectable HI activity, emitted 3-Hal as the sole GLV, whereas transgenic tomato plants that overexpressed paprika $\mathrm{HI}$ emitted 2-Hal as the sole GLV, thereby indicating that the presence of HI determines whether 3-Hal or 2-Hal is emitted from wounded plants. ${ }^{4}$ Furthermore, the emission of 2-Hal from HI-overexpressing tomato plants was rapid, which is consistent with the fact that $\mathrm{HI}$ does not require cofactors to produce 2-Hal, as well as lipase, lipoxygenase, and hydroperoxide lyase.

2 -Hal is much more chemically reactive than other GLVs because it possesses an $\alpha, \beta$-unsaturated carbonyl bond, and this chemical property contributes to 2-Hal's physiological role. Indeed, we previously reported that $\mathrm{C} 4-\mathrm{C} 9$ straight-chain carbonyls with $\alpha, \beta$-unsaturated carbonyl bonds (i.e., named reactive short-chain leaf volatiles, RSLVs) can function as signaling chemicals that induce heat- and oxidative stress-responsive gene expression. ${ }^{17)}$ Furthermore, 2 - Hal is the only RSLV that is biosynthesized by a series of enzymes, ${ }^{4}$ rather than through nonenzymatic mechanisms. ${ }^{17)}$

In agreement with the NimbleGen array data, the present study's data, which was obtained using an Agilent array, indicated that 2-Hal strongly induces the expression of genes related to heat and oxidative stress responses (Fig. 2). The numbers of genes up- and downregulated by 2-Hal (1419 and 2649, respec- tively) were the lowest among the GLVs tested (Fig. 5A). Corresponding to our previous study, important transcription factors for the regulation of gene expression for each stress (HSFA2, $D R E B 2 A$, and MBF1c for heat stress; ZATs for oxidative stress) were listed among the top 100 upregulated genes (Fig. 5B). 2-Hal also upregulated defense-responsible genes, but the number of defense genes was fewer than the number of $(Z)$-3-series GLVs. In contrast to the other GLVs, 2-Hal plays an important role in inducing oxidative stress responses, which suggests that RSLVs, including 2-Hal, are abiotic stress-related infochemicals, and, thus, 2-Hal emission is enhanced by abiotic stresses such as heat ${ }^{26)}$ and drought. ${ }^{27)}$

On the contrary, gene expression data obtained using the Agilent array indicated that 2-Hal downregulated a group of genes related to auxin metabolism, possibly accounting for the ability of 2-Hal to inhibit root elongation. ${ }^{28)}$

4. Effects of 3-hexenyl acetate (3-HAC, 4) on gene expression Because 3-HAC is oriented downstream of the GLV biosynthesis pathway (Fig. 1), 3-HAC is the last GLV emitted after wounding. In addition, 3-HAC is emitted from intact cells, ${ }^{5}$ since its biosynthesis requires $\mathrm{CHAT}$, acetyl-CoA, and $3-\mathrm{Hol}$ as substrates.

In the seedlings of $A$. thaliana treated with 3-HAC for $30 \mathrm{~min}$, more genes were upregulated $(n=6098)$ than downregulated $(n=1883$; Fig. 6A), and a number of defense and heat-shock response genes were included among the top 100 upregulated genes (Fig. 6B). As in the 3-Hal and 3-Hol treatments, PR2 (ranked 22nd) was upregulated. 3-HAC induced SA-responsible defense response genes such as PR1 and chitinase, indicat-

(A)

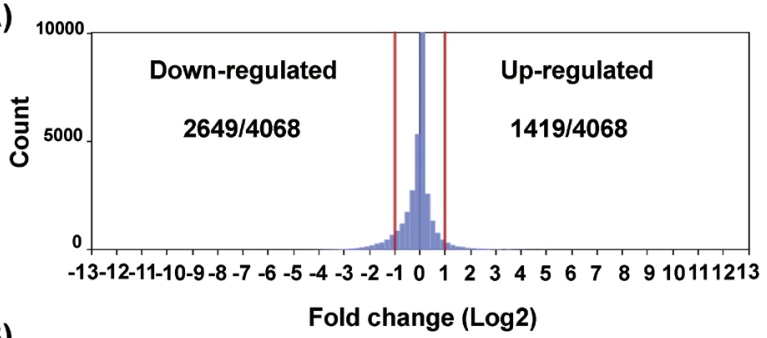

(B)

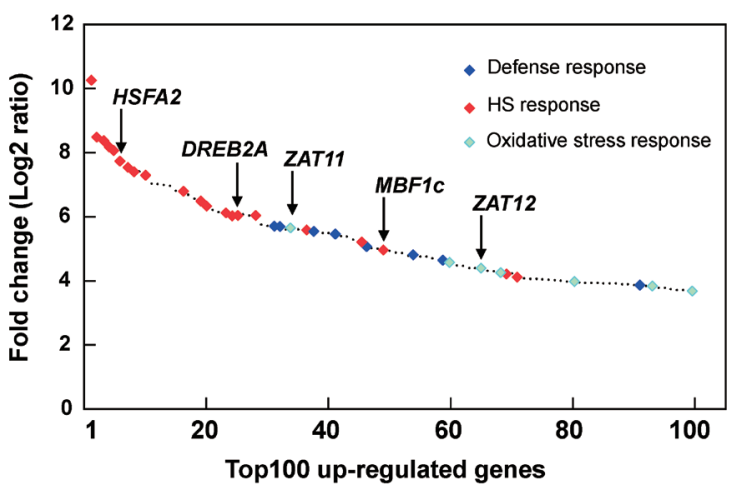

Fig. 5. Effect of 2-Hal on gene expression in Arabidopsis. Legends of panels (A) and (B) are the same as those for Fig. 3, in which the light blue triangles indicate oxidative stress response genes. A detailed list is shown in Table S3. 
(A)

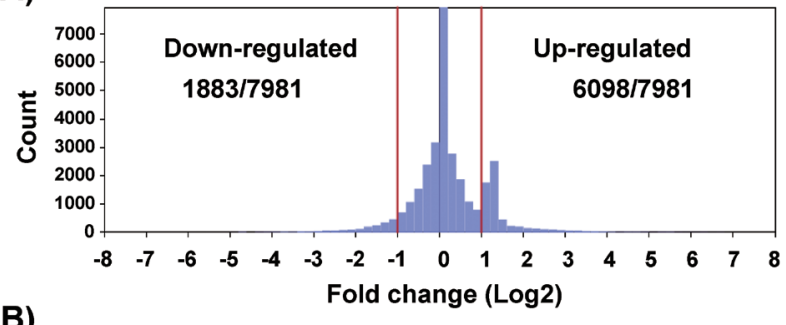

(B)

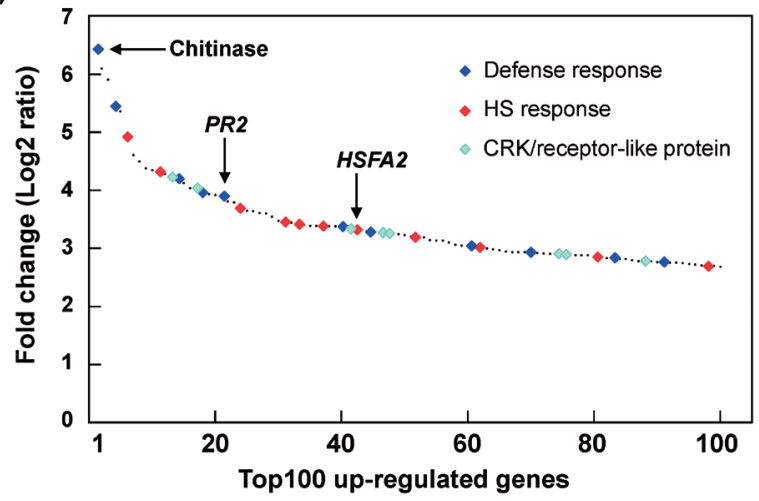

Fig. 6. Effect of 3-HAC on gene expression in Arabidopsis. Legends of panels (A) and (B) are the same as those for Fig. 3. A detailed list is shown in Table S4.

ing that 3-HAC can activate SA-responsible defense responses. Meanwhile, 3-HAC downregulated cold stress-responsive genes (Fig. 2B); however, the physiological significance of this change is unknown.

Exogenous 3-HAC has been reported to prime plants against pathogens and insect attacks. For example, 3-HAC-treated wheat exhibited greater resistance to Fusarium graminearum, ${ }^{19)}$ owing to the induction of SA-responsive genes, and in corn, 3-Hal, 3-Hol, and 3-HAC exhibited nearly identical priming activity against insect attack, ${ }^{18)}$ which corresponds to the finding of the present study that (Z)-3 series GLVs commonly induce the expression of defense response genes. Especially, PRRs upregulated by the $(Z)$-3-series GLVs might contribute to resistance against enemy attack.

\section{Spatiotemporal effects of GLVs in wounded Arabidopsis}

Comprehensive transcriptome analysis indicated that major GLVs have specific gene expression spectra, and the major gene groups induced by GLVs are defense response genes. Recently, GLVs have been considered damage-associated molecular patterns (DAMPs) that carry specific information about herbivores and pathogens. ${ }^{20)}$ Data from the present study also support the idea that GLVs can act as self-made DAMPs when plants are wounded. In this context, the $\beta, \gamma$-unsaturated bond in the (Z)-3-series GLVs might be a sign for triggering the defense response because 3-Hal, 3-Hol, and 3-HAC are commonly stimulated defense responses. On the contrary, the $\alpha, \beta$-unsaturated carbonyl bond in 2-Hal might be a sign for triggering an oxidative stress response because $\mathrm{C} 4-\mathrm{C} 9$ straight-chain compounds that have an $\alpha, \beta$-unsaturated carbonyl bond exhibit identical activity.

As shown in Fig. 1, the endogenous substrate of GLVs is linolenic acid from the thylakoid membrane. When leaves are damaged, linolenic acid becomes accessible because of the disintegration of thylakoid membranes in damaged cells." ${ }^{3)}$ Therefore, GLV is primarily produced in the damaged cells and, secondarily, in nearby intact cells (Fig. 7). $3-\mathrm{Hal}$ and 2 -Hal are produced in the damaged cells by a series of enzymes that do not require the presence of cofactors. Production is then expanded to neighboring intact cells, where 3-Hal diffused from damaged cells is used as a precursor for 3-Hol and 3-HAC production. On the contrary, GLV acceptor sites are the intact cells around damaged cells; therefore, the production and acceptor sites of 3-Hol and 3-HAC might be identical. Together with the transcriptome data obtained in the present study, the scenario for the biological impact of GLVs emitted after wounding can be hypothesized as described below.

When a leaf is wounded, the first GLV, 3-Hal, is immediately emitted by the damaged cells, and in plant species with high $\mathrm{HI}$ activity, $2-\mathrm{Hal}$ is produced immediately after 3 -Hal production. 3-Hal and 2-Hal are primarily produced at injury sites, where they function as antibiotics for direct defense. They then diffuse to nearby intact cells, where 3-Hal activates defense responses and downregulates cell cycle processes, and 2-Hal helps sustain protein homeostasis and oxidative stress response as an airborne infochemical. 3-Hal diffused from the damaged cells functions both as an infochemical and as a substrate for subsequent GLV production. In other words, 3-Hal is converted to 3-Hol in nearby intact cells, and 3-Hol functions in a manner similar to 3-Hal. 3 -Hol is further processed to 3-HAC, which functions as an in-

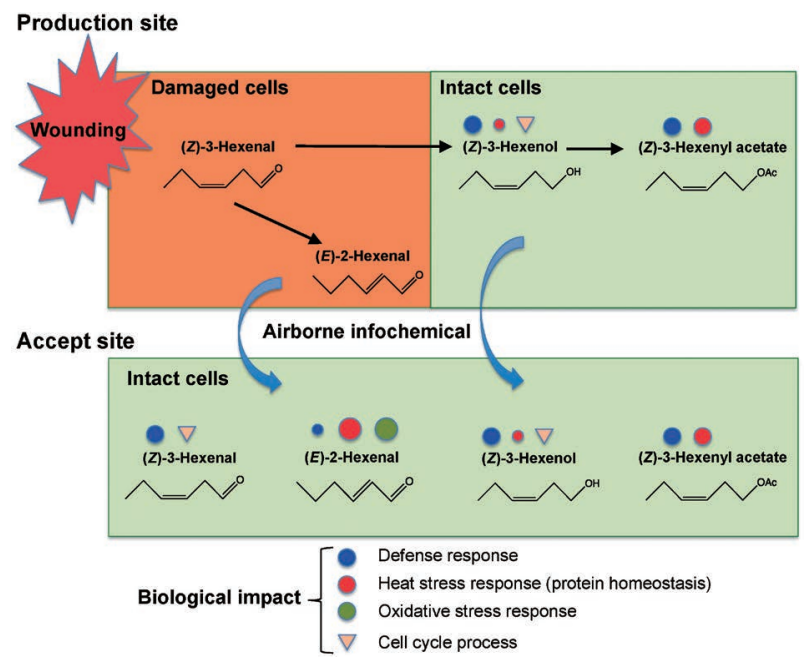

Fig. 7. Hypothesized model of GLV production and functions. 3-Hal and 2-Hal are produced by damaged cells, whereas 3-Hol and 3-HAC are produced by adjacent intact cells, using diffused 3-Hal as a substrate. Airborne GLVs are accepted by intact cells and alter gene expression. Circles and triangles indicate activated and inactivated biological processes, respectively. 
fochemical that induces both a defense response and protein homeostasis.

Consequently, the sequential production of GLVs at different sites contributes to the diverse spatiotemporal effect of GLVs in wounded plants. In the present study, we used individual GLVs for experiments. However, the GLVs naturally emitted by wounded leaves are blended and, therefore, have complex biological effects. Because the expression patterns of GLVs do not interfere with one another (Fig. 2B), we speculate that the effect of GLVs might be additive. Therefore, the effect of blended GLVs on gene expression must be analyzed in the future.

\section{Acknowledgements}

This work was supported in part by JSPS KAKENHI (grant no. 26740015).

\section{References}

1) Ü. Niinemets: Trends Plant Sci. 15, 145-153 (2010).

2) K. Matsui: Curr. Opin. Plant Biol. 9, 274-280 (2006).

3) M. Ameye, S. Allman, J. Verwaeren, G. Smagghe, G. Haesaert, R. C. Schuurink and K. Audenaert: New Phytol., doi: 10.1111/nph.14671 (2017).

4) M. Kunishima, Y. Yamauchi, M. Mizutani, M. Kuse, H. Takikawa and Y. Sugimoto: J. Biol. Chem. 291, 14023-14033 (2016).

5) K. Matsui, K. Sugimoto, J. Mano, R. Ozawa and J. Takabayashi: PLoS One 7, e36433 (2012).

6) J. C. D’Auria, E. Pichersky, A. Schaub, A. Hansel and J. Gershenzon: Plant J. 49, 194-207 (2007).

7) R. Fall, T. Karl, A. Hansel, A. Jordan and W. Lindinger: J. Geophys. Res. 104(D13), 15963-15974 (1999).

8) S. Nakamura and A. Hatanaka: J. Agric. Food Chem. 50, 7639-7644 (2002).

9) K. Kishimoto, K. Matsui, R. Ozawa and J. Takabayashi: Phytochemis$\operatorname{try}$ 69, 2127-2132 (2008).

10) D. G. Hammond, S. Rangel and I. Kubo: J. Agric. Food Chem. 48, 4410-4417 (2000).

11) J. Hubert, Z. Münzbergová and A. Santino: Pest Manag. Sci. 64, 57-
64 (2008)

12) J. H. Visser, S. van Straten and H. Maarse: J. Chem. Ecol. 5, 13-25 (1979).

13) T. C. J. Turlings, J. H. Tumlinson, R. R. Heath, A. T. Proveaux and R. E. Doolittle: J. Chem. Ecol. 17, 2235-2251 (1991).

14) A. Scala, S. Allmann, R. Mirabella, M. A. Haring and R. C. Schuurink: Int. J. Mol. Sci. 14, 17781-17811 (2013).

15) K. Sugimoto, K. Matsui, Y. Iijima, Y. Akakabe, S. Muramoto, R. Ozawa, M. Uefune, R. Sasaki, K. M. Alamgir, S. Akitake, T. Nobuke, I. Galis, K. Aoki, D. Shibata and J. Takabayashi: Proc. Natl. Acad. Sci. U.S.A. 111, 7144-7149 (2014).

16) K. Kishimoto, K. Matsui, R. Ozawa and J. Takabayashi: Plant Cell Physiol. 46, 1093-1102 (2005).

17) Y. Yamauchi, M. Kunishima, M. Mizutani and Y. Sugimoto: Sci. Rep. 5, 8030 (2015).

18) J. Engelberth, H. T. Alborn, E. A. Schmelz and J. H. Tumlinson: Proc. Natl. Acad. Sci. U.S.A. 101, 1781-1785 (2004).

19) M. Ameye, K. Audenaert, N. De Zutter, K. Steppe, L. Van Meulebroek, L. Vanhaecke, D. De Vleesschauwer, G. Haesaert and G. Smagghe: Plant Physiol. 167, 1671-1684 (2015).

20) D. Duran-Flores and M. Heil: Curr. Opin. Plant Biol. 32, 77-87 (2016).

21) J. Kilian, D. Whitehead, J. Horak, D. Wanke, S. Weinl, O. Batistic, C. D’Angelo, E. Bornberg-Bauer, J. Kudla and K. Harter: Plant J. 50, 347-363 (2007).

22) D. W. Huang, B. T. Sherman and R. A. Lempicki: Nat. Protoc. 4, 4457 (2009).

23) Z. Chen: Plant Physiol. 126, 473-476 (2001).

24) Y.-H. Yeh, Y.-H. Chang, P.-Y. Huang, J.-B. Huang and L. Zimmerli: Front. Plant Sci. 6, 322 (2015).

25) C. Zipfel: Trends Immunol. 35, 345-351 (2014).

26) F. Loreto, C. Barta, F. Brilli and I. Nogues: Plant Cell Environ. 29, 1820-1828 (2006).

27) S. Catola, G. Marino, G. Emiliani, T. Huseynova, M. Musayv, Z. Akparov and B. E. Maserti: Planta 243, 441-449 (2016).

28) R. Mirabella, H. Rauwerda, E. A. Struys, C. Jakobs, C. Triantaphylides, M. A. Haring and R. C. Schuurink: Plant J. 53, 197-213 (2008). 